\title{
Экстракционно-хроматографическое выделение неодима из азотнокислых растворов с применением сорбентов, импрегнированных $\mathbf{N}, \mathbf{N}, \mathbf{N}, \mathbf{N}^{\prime}$-тетраоктилдигликольамидом
}

\author{
Баулин В.Е. ${ }^{1,2}$, Баулин Д.В. ${ }^{2}$, Усолкин А.Н. ${ }^{3}$, Ивенская Н.М., \\ Власова Н.В. ${ }^{3}$, Козлов П.В. ${ }^{3}$, Ремизов М.Б. ${ }^{3}$, \\ Чухланцева Е. В. ${ }^{3}$, Цивадзе А.Ю. ${ }^{2}$ \\ ${ }^{1}$ ФББУН Институт физической химии и электрохимии имени А.Н. Фрумкина, Москва \\ ${ }^{2}$ ФГБУН Институт физиологически активных веществ РАН, Черноголовка \\ ${ }^{3}$ ФГУП «ПО Маяк», Озерск
}

Поступила в редакцию 5.03.2018 г.

DOI: https://doi.org/10.17308/sorpchrom.2018.18/598

Исследованы особенности экстракционно-хроматографического выделения неодима как аналога америция из азотнокислых растворов РЗЭ сорбентами, импрегнированными N,N,N',N'тетраоктилдигликольамидом (ТОДГА). Определен оптимальный количественный и качественный состав сорбента, условия сорбции и десорбции, обеспечивающие наиболее эффективное извлечение неодима. Установлено, что наибольшими перспективами практического применения обладает сорбент, в котором в качестве матрицы используется гидрофобизированный силикагель.

Ключевые слова: экстракционно-хроматографическое выделение, азотнокислые растворы, неодим, америций, лантан, европий, самарий, N,N,N',N'- тетраоктилдигликольамид, сорбенты импрегнированного типа, сополимеры стирола с дивинилбензолом, гидрофобизированный силикагель

\section{The extraction-chromatographic separation of neodymium from solution from nitric acid with application of sorbents impregnated by $\mathbf{N}, \mathbf{N}, \mathbf{N}$,,N'-tetraoctyldiglicolamide}

\author{
Baulin V.E. ${ }^{1,2}$, Baulin D.V. ${ }^{1}$, Usolkin A.N. ${ }^{3}$, Ivenskaya N.M. ${ }^{3}$, Vlasova N.V. ${ }^{3}$, \\ Kozlov P.V. ${ }^{3}$, Remizov M.B. ${ }^{3}$, Chuchlanseva E.V. ${ }^{3}$, Tsivadze A.Y. ${ }^{1}$ \\ ${ }^{I}$ Frumkin Institutde of Physical Chemistry and Electrochemistry, Russian Academy of Sciences, Moscow \\ ${ }^{2}$ Institute of Physiologicall Active Substances, Russian Academy of Sciences, Chernogolovka \\ ${ }^{3}$ PO Mayak, Ozyorsk, Chelyabinsk oblast
}

The purpose of this work is investigation of extraction-chromatographic separation of neodymium, as analogue of americium from nitrate solutions of some rare-earth elements (La(III), Eu(III) и Sm(III) with application of sorbents impregnated by $\mathrm{N}, \mathrm{N}, \mathrm{N}^{\prime}, \mathrm{N}^{\prime}$ - tetraoctyldiglicolamide (TODGA). The laboratory and commercial accessible patterns of sorbents, which differences by the method of synthesis, content of TODGA, size of granule and nature matrice were investigated. The laboratorial method of the receiving of sorbents impregnated by TODGA was described. The standard test solutions of Am(III), Nd(III), La(III), Eu(III) 
и $\mathrm{Sm}$ (III definite concentration was used in present investigations. The determination of concentrations of neodymium in investigated solutions was performed by spectrophotometric method with utilization reagents of Arsenazo M $(\lambda=660 \mathrm{HM})$ or personal light absorption of neodymium ( $\lambda=796 \mathrm{HM})$, depending on concentrations of neodymium in solutions. The investigations were conducted in frontal and eluted regimes. The significances of dynamic coefficient distribution for neodymium were obtained by means of the standard treatment of frontal dynamic curves. The eluted curves were obtained by the way of desorption of elements after preliminary mapping of definite quantity of analyzed solution on the chromatographic column with the sorbent.

The solutions of $0.05 \mathrm{M} \mathrm{HNO}_{3}$, the mixture solutions of $0.05 \mathrm{M} \mathrm{HNO}_{3}$ and $\mathrm{H}_{3} \mathrm{PO}_{4}(0.01 \mathrm{M}$ и $0.1 \mathrm{M})$, the mixture of solutions of $0.05 \mathrm{M} \mathrm{HNO}_{3}$ and $0.01 \mathrm{M}$ diethylenetriaminepentaacetic acid (DTPA) were investigated as the desorption agents .

The value of the dynamic coefficients of distribution for neodymium, according to its concentration in the stock solutions, eluent flow rate and size of sorbents granule were received. The distinctive features of extraction-chromatographic separation of neodymium, as analogue of americium from nitrate solutions of rare-earth elements with application of sorbents impregnated by TODGA) were investigated. The relevancy of utilization of $\mathrm{Nd}(\mathrm{III})$ in quality analogue of $\mathrm{Am}$ (III) was certified by the way of practical identity of the frontal curve for neodymium and americium, which were obtained in identical conditions.

The optimal qualitative and quantitative compositions of sorbents and conditions for effective sorption and desorption of neodymium were found experimentally. It is established that the sorbents wherein as matrices applied of hydrophobic silica gel, possess the greatest opportunity for practical application. In addition, in question sorbent be able selective extract of lanthanum, europium and samarium, that permit of possibility applications for intragroup division of this rare-earth elements.

Keywords: extraction chromatography, neodymium, americium, lanthanum, europium, samarium, $\mathrm{N}, \mathrm{N}, \mathrm{N}^{\prime}, \mathrm{N}^{\prime}$ - tetraoctyldiglicolamide, sorbents impregnated type, copolimer of styrene and divinyl benzene, hydrophobic silica gel.

\section{Введение}

Проблема разделения лантанидов и минорных актинидов (Am, Np, Cm) является одной из важных задач в создании замкнутого ядерного топливного цикла. Минорные актиниды, в частности америций, обладают высокой удельной радиоактивностью, поэтому предварительные эксперименты по его выделению целесообразно проводить на стабильных препаратах $\mathrm{Nd}(\mathrm{III})$, который по ряду физических и химических свойств, является аналогом Аm(III).

Для разделения близких по химическим свойствам элементов большое распространение получили экстракционные методы [1]. Однако, в последнее время, наметилась устойчивая тенденция предпочтительного использования сорбционных технологий, что обусловлено как высокой эффективностью и избирательностью извлечения, так и простотой выполнения операций с радиоактивными образцами [2].

Для практической реализации сорбционных технологий необходимо располагать эффективными сорбционными материалами (сорбентами). Среди известных сорбентов определенный интерес представляют технологически доступные сорбенты импрегнированного типа, которые изготовляются путем нековалентного закрепления (импрегнации) органических экстрагентов на органические (сополимеры стирола с дивинилбензолом) или неорганические макропористые носители. Сорбционные характеристики сорбентов импрегнированного типа (коэффициенты извлечения и селективности, емкость по извлекаемому компоненту, количество рабочих циклов сорбция/десорбция и т.д.) определяются как свойствами органического экстрагента и носителя, так и физико-химическими особенностями супрамакромолекулярной организации поверхностного слоя неподвижной фазы на поверхности носителя.

$\mathrm{N}, \mathrm{N}, \mathrm{N}, \mathrm{N}^{\prime}-$ тетраоктилдигликольамид (ТОДГА) (рис.1), по-видимому, является одним из наиболее перспективных экстрагентов для разделения лантанидов и актинидов из азотнокислых растворов [3], ТОДГА применяется в качестве компонента неподвижной фазы в коммерчески доступном сорбенте импрегнированного типа 
«DGA Resin». Сорбент «DGA Resin» успешно применяется для разделения Р3Э и TПЭ, Sr/Y, Ra/Ac и для решения ряда других радиохимических задач [4-6]. Описаны результаты исследований возможностей применения импрегнированных сорбентов на основе ТОДГА для отделения макроколичеств америция от сопутствующих примесей [7], а в [8] исследована сорбция Р3Э(III), Th(IV) и U(VI).

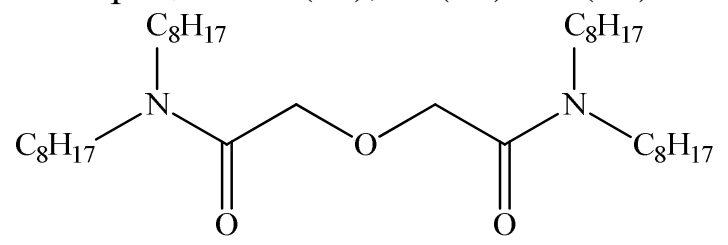

Рис. 1. Структурная формула N,N,N',N'- тетраоктилдигликольамида

В настоящей работе исследовано экстракционно-хроматографическое выделение $\mathrm{Nd}(\mathrm{III})$ из азотнокислых растворов с применением как полученных самостоятельно в лабораторных условиях, так ряда коммерчески доступных сорбентов, импрегнированных ТОДГА, марок AXIONIT MND 40T, BAU-1M и SBU-3, изготовленных отечественными производителями, различающихся массовым содержанием ТОДГА, технологиями изготовления, гранулометрическим составом и материалом макропористого носителя. Получены зависимости динамических коэффициентов распределения неодима от его концентрации в исходном растворе и величины расхода элюента. Определены составы растворов, обеспечивающие эффективную десорбцию неодима. Также исследована сорбция неодима и его ближайших аналогов лантана, европия и самария.

\section{Эксперимент}

Для получения лабораторных образцов импрегнированных сорбентов использовали $\mathrm{N}, \mathrm{N}, \mathrm{N}$, $\mathrm{N}^{\prime}$-тетраоктилдигликольамид с содержанием основного компонента не менее 98\% производства ООО «Сорбент-Технологии» (г. Москва). В качестве носителя использовали сополимер стирола с дивинилбензолом «LPS-500» (размер фракций 40-70, 50-100, 100-250, 150-315 мкм) производства ООО «Техносорбент» г. Москва.

Лабораторная методика получения сорбента, импрегнированного 20 \% ТОДГА. В стакан емкостью $250 \mathrm{~cm}^{3}$ помещали раствор 2.5 г ТОДГА в $50 \mathrm{~cm}^{3}$ хлороформа (ч.д.а.), затем добавляли 10 г гранулированного полимера «LPS-500». Полученную смесь тщательно перемешивали и сушили при комнатной температуре в вытяжном шкафу до сыпучего состояния (8-10 ч). Выход сорбента составил 12.5 г (100\%). Сорбенты, различающиеся массовой долей ТОДГА, размером гранул и природой носителя, получали аналогичным образом.

В работе исследовался и ряд коммерчески доступных сорбентов, изготовленных на основе ТОДГА: AXIONIT MND 40T - сорбент производства ЗАО «АксионРДМ» (г. Пермь), полученный путем введения ТОДГА на стадии сополимеризации стирола с дивинилбензолом. Сорбент представляет собой сферические гранулы бе-

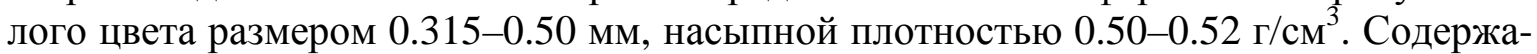
ние ТОДГА $40 \%$ масс.

BAU-1M - сорбент производства ООО «Сорбент-Технологии» (Москва), полученный путем импрегнирования коммерчески-доступного носителя - сополимера стирола с дивинилбензолом марки MN-202 («Purolite», Великобритания). Содержание ТОДГА в сорбенте составляет $50 \%$ масс. Сорбент представляет собой сфериче- 
ские гранулы темно-коричневого цвета размером 0.4-0.6 мм, насыпной плотностью $0.43-0.44 \mathrm{r} / \mathrm{cm}^{3}$.

SBU-3 - сорбент производства ООО «Сорбент-Технологии» (г. Москва), полученный путем импрегнирования носителя - гидрофобизированного силикагеля марки КСКГ. Сорбент представляет собой гранулы белого или кремового цвета раз-

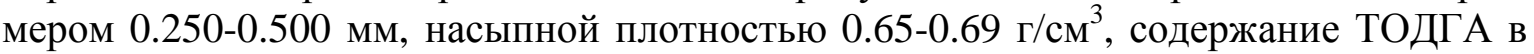
сорбенте 30-33 \% масс. Для проведения исследований использовали модельные растворы Am(III), Nd(III), La(III), Eu(III) и Sm(III) известных концентраций.

Сорбцию неодима в динамических условиях проводили путем пропускания исходного раствора через стеклянную колонку с внутренним диаметром 8 мм, заполненную сорбентом в набухшем состоянии. В связи с тем, что сорбенты на основе ТОДГА имеют гидрофобную поверхность и всплывают в водных растворах, слой сорбента в колонке сверху и снизу фиксировали кусочками стеклоткани. При изучении сорбентов мелких фракций раствор пропускали через колонку сверху вниз с постоянной скоростью при помощи перистальтического насоса, в случае сорбентов более крупных фракций эксперименты проводились в самотечном режиме. Фильтрат после колонки собирали по фракциям и анализировали на содержание поглощаемого компонента. Определение концентрации неодима в растворе на выходе из колонки проводили спектрофотометрическим методом при соотношении $\mathrm{V}_{\text {.p-pa }}: \mathrm{V}_{\text {реагента }}=1: 2$, c использованием реагента арсеназо-М, который образует окрашенный комплекс с неодимом в широком диапазоне концентраций азотной кислоты с максимумом поглощения при длине волны 650 нм., либо по собственному светопоглощению неодима $\left(\lambda_{\text {макс. }}=796\right.$ нм $)$, в зависимости от исходной концентрации неодима в растворе.

Колонки заполняли водной суспензией сорбента, которую предварительно дегазировали под вакуумом. Значения динамических коэффициентов распределения рассчитывали как отношение объёма раствора, который может быть профильтрован через слой сорбента до момента «проскока» в фильтрат определённого количества извлекаемого вещества, к массе сорбента [9]. В качестве «проскоковой» концентрации элемента, использовалась «половинная» концентрация.

Элютивные выходные кривые получали путем десорбции сорбированных элементов после предварительного нанесения определенного количества исследуемого раствора на колонку, заполненную сорбентом. Для десорбции использовали следующие растворы: $0.05 \mathrm{M} \mathrm{HNO}_{3}$; смесь $0.05 \mathrm{M} \mathrm{HNO}_{3}$ и $\mathrm{H}_{3} \mathrm{PO}_{4}$ с концентрацией последней 0.01 и 0.1 моль/дм ; смесь $0.05 \mathrm{M} \mathrm{HNO}_{3}$ и $0.01 \mathrm{M}$ ДТПА.

На выходе из колонки собирали фракции элюата по $3 \mathrm{~cm}^{3}$, в которых измеряли оптическую плотность, согласно которой определяли эффективность десорбции сорбированных элементов после их сорбции из растворов с различной концентрацией $\mathrm{HNO}_{3}$. Объём раствора, необходимый для полной десорбции элемента с сорбента, определялся как разница объёмов с момента появления элемента в элюате и до его практически полного исчезновения.

\section{Обсуждение результатов}

В настоящей работе $\mathrm{Nd}(\mathrm{III})$ использовали в качестве аналога $\mathrm{Am}(\mathrm{III})$. Правомерность такого подхода подтверждается практической идентичностью полученных нами в одинаковых условиях фронтальных выходных кривых для неодима и америция (рис. 2). На рисунке 3 представлены фронтальные выходные кривые сорбции неодима в зависимости от скорости пропускания раствора. Установлено, что с увеличением скорости пропускания исходного раствора коэффициент распределения неодима уменьшается, что, по-видимому, обусловлено недостаточной скоростью про-

Баулин и др. / Сорбционные и хроматографические процессы. 2018. Т. 18. № 5 
хождения массообменных процессов. Коэффициенты распределения неодима при расходе элюента 6 к.о./мин и 3 к.о./мин различаются незначительно, а время проведения эксперимента при расходе 6 к.о./мин уменьшается в два раза. Следовательно, наиболее приемлемым является расход исходного раствора, равный 6 к.о./мин.

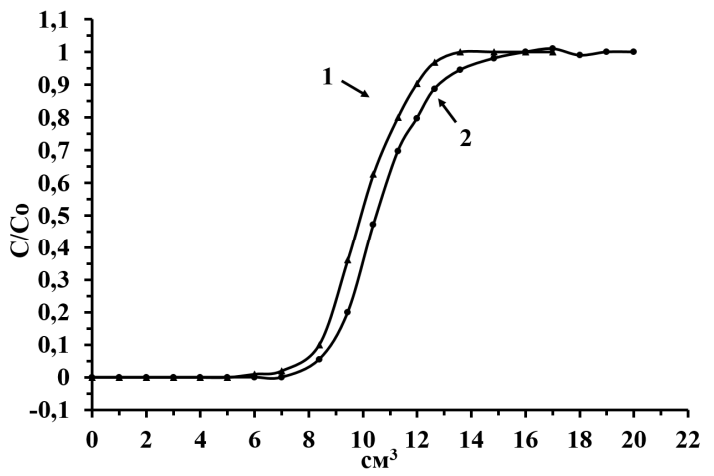

Рис. 2. Фронтальные выходные кривые сорбции неодима (1) и америция (2) из 3 М азотной кислоты. Сорбент: $50 \%$

ТОДГА, носитель LPS-500; фракция:

50-100 мкм; масса сорбента в колонке: 30 мг; расход исходного раствора: $1 \mathrm{~cm}^{3} /$ мин; концентрация неодима и америция $1.4 \mathrm{r} / \mathrm{cm}^{3}$

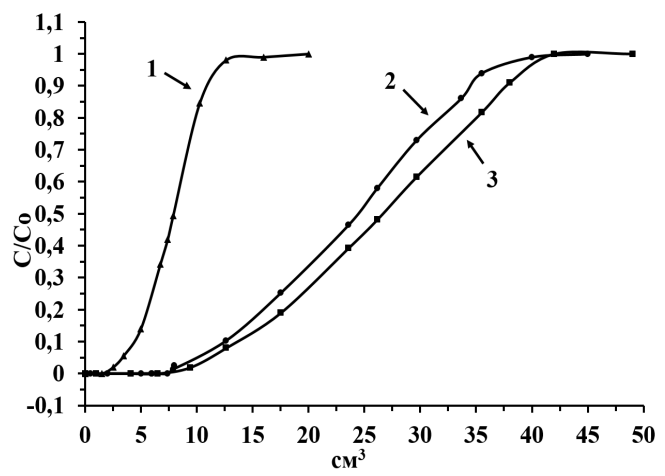

Рис. 3. Фронтальные выходные кривые сорбции неодима из $2 \mathrm{M}$ азотной кислоты в зависимости от расхода элюента. Сорбент: 23\% ТОДГА LPS-500, фракция: 50-100 мкм; масса сорбента в колонке: 40 мг; массовая концентрация неодима: 5 мг/дм³ ; расход исходного раствора: расход исходного раствора: $1-2 \mathrm{~cm}^{3} / \mathrm{мин;}$

\section{$2-0.5 \mathrm{~cm}^{3} /$ мин; $3-0.25 \mathrm{~cm}^{3} /$ мин}

Влияние гранулометрического состава сорбентов на сорбционное извлечение неодима весьма велико - значения динамических коэффициентов распределения для неодима на сорбенте с разным размером гранул составили $7.3 \cdot 10^{2}, 9.7 \cdot 10^{2}, 1.7 \cdot 10^{3}$ (соответственно кривые 1, 2 и 3, рис.4), то есть наиболее эффективным является сорбент с самым меньшим размером гранул и наиболее узкой фракции (рис.4).

Увеличение массовой доли ТОДГА с 20 до 50 \% закономерно приводит к увеличению коэффициентов распределения неодима и, соответственно, к более эффективному процессу его сорбции. Значения динамических коэффициентов распределения неодима составляют $8 \cdot 10^{2}, 1.7 \cdot 10^{3}, 4 \cdot 10^{3}$ для кривых $1,2,3$, соответственно (рис. 5).

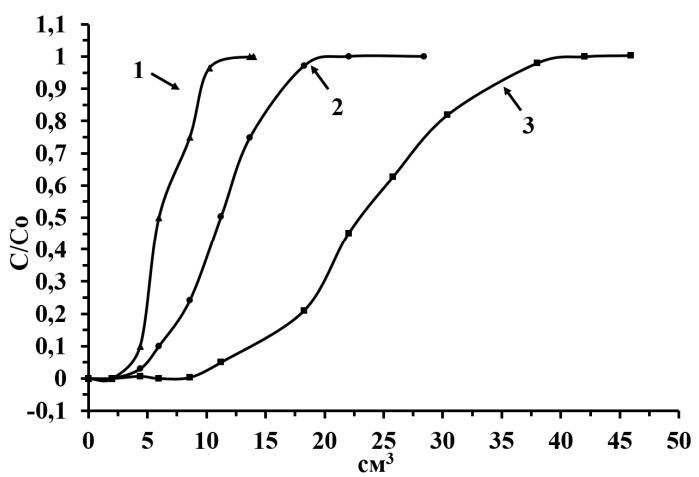

Рис. 4. Выходные кривые сорбции неодима из 2 М азотной кислоты сорбентами с различным размером гранул. Сорбент: $33 \%$ ТОДГА LPS-500; масса сорбента в колонке: 40 мг; массовая концентрация неодима: 55 мг/дм 3 ; расход исходного раствора: $0.5 \mathrm{~cm}^{3}$ / мин. 1 - 150-300 мкм; 2 - 100-250 мкм; 3- 4070 мкм

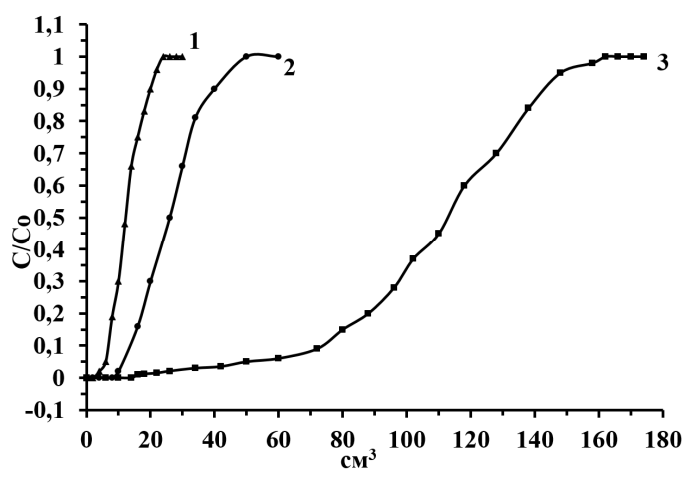

Рис. 5. Выходные кривые сорбции неодима из $2 \mathrm{M}$ азотной кислоты в зависимости от массового содержания экстрагента на матрице LPS-500. Фракция: 40-70 мкм; масса сорбента в колонке: 40 мг; массовая концентрация неодима: 5 мг/дм ${ }^{3}$; расход исходного раствора: $0.5 \mathrm{~cm}^{3} /$ мин Массовая доля ТОДГА для кривых 1,2 и 3 , соответственно, 20 , 
В экспериментах по десорбции показано, что промывка колонки $0.05 \mathrm{M} \mathrm{HNO}_{3}$ приводит к полной десорбции неодима, при этом добавление к десорбирующему раствору как $\mathrm{H}_{3} \mathrm{PO}_{4}$, так и ДТПА не оказывает существенного влияния на эффективность десорбции в условиях проведения данного эксперимента. Объёмы десорбирующих растворов, независимо от их состава, составляют примерно от 15 до $25 \mathrm{~cm}^{3}$ (рис.6).
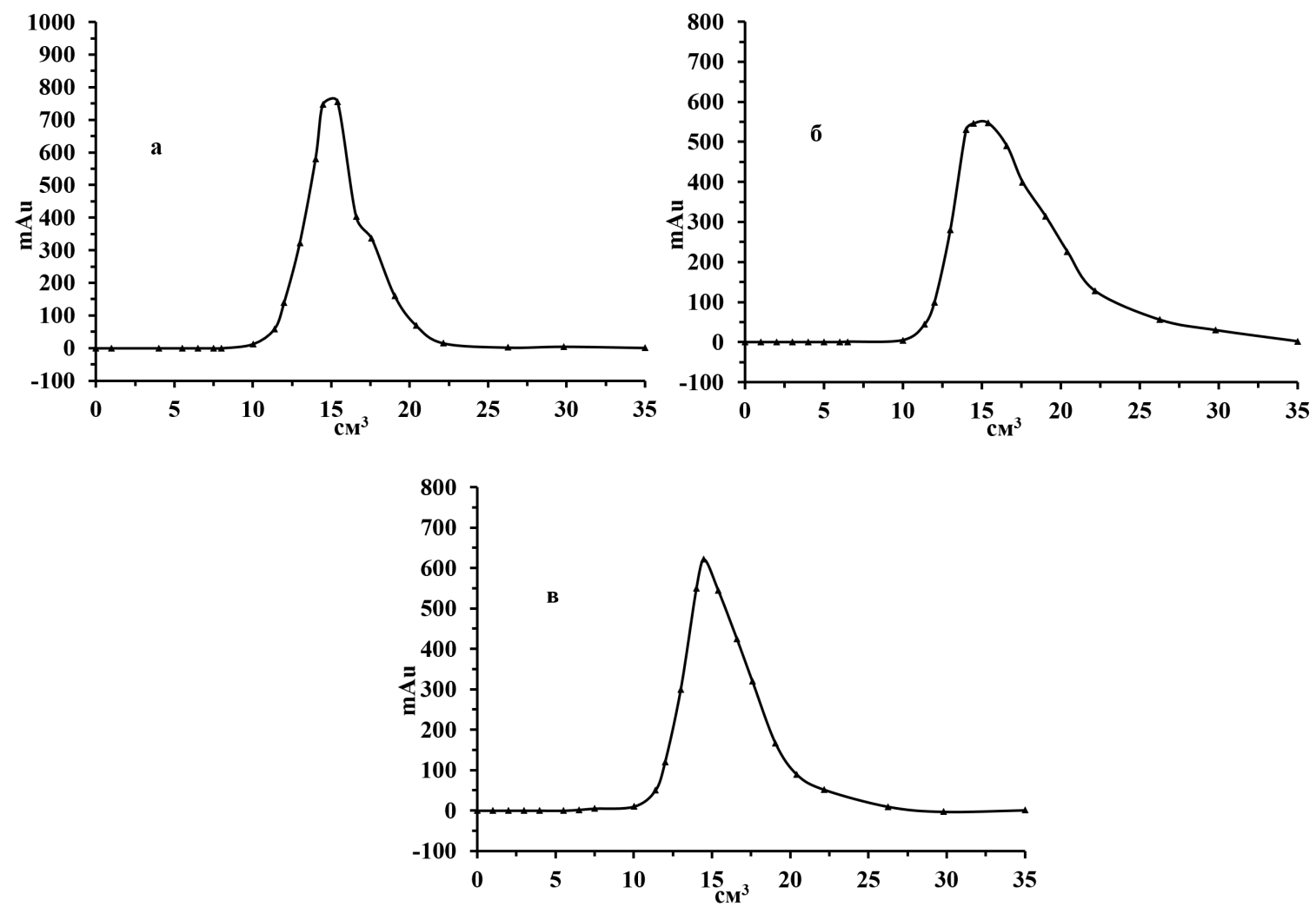

Рис. 6. Хроматограмма десорбции неодима после сорбции из $5 \mathrm{M} \mathrm{HNO}_{3}$ с сорбента ТОДГА (фракция: 40-70 мкм; масса сорбента в колонке: 40 мг; массовая концентрация неодима при сорбции : 50 мг/дм³; расход исходного раствора: $0.5 \mathrm{~cm}^{3}$ / мин) десорбирующими растворами различного состава. а - $0.05 \mathrm{M} \mathrm{HNO}_{3}+0.01 \mathrm{M}$ $\mathrm{H}_{3} \mathrm{PO}_{4}$. б - $0.05 \mathrm{M} \mathrm{HNO}_{3}$. в. $0.05 \mathrm{M} \mathrm{HNO}_{3}+0.01$ г/л ДТПА

Таким образом, сорбенты импрегнированного типа на основе ТОДГА в качестве экстрагента и полимера стирола с дивинилбензолом марки LPS-500 в качестве носителя весьма эффективно могут применяться для извлечения неодима из азотнокислых растворов. Однако следует отметить, что используемые в экспериментах сорбенты достаточно мелких фракций (40-70 и 100-250 мкм), не применимы в технологии, так как их использование в колонках большого размера закономерно приведёт к созданию в системе повышенного давления вследствие высокого гидравлического сопротивления слоя сорбента. Поэтому нами была исследована возможность выделения неодима на коммерчески доступных сорбентах более крупных фракций, изготовленных на основе ТОДГА и сополимеров стирола с дивинилбензолом BAU$1 \mathrm{M}$ и AXIONIT MND 40T [8] и нового сорбента на основе ТОДГА марки SBU-3, в котором в качестве матрицы используется гидрофобизированный силикагель отечественного производства. Последний изготавливается путем обработки калиброванного силикагеля марки КСКГ триметилхлорсиланом, что гарантирует как заранее определенный гранулометрический состав, так и высокие гидрофобные свойства. Более того, сорбент SBU-3 характеризуется пониженным содержанием достаточно 
дорогого экстрагента ТОДГА (33\%). Несомненным достоинством сорбента SBU-3 является и способность растворяться в растворах щелочей, что обеспечивает эффективное извлечение загрязненного радиоактивными изотопами отработавшего сорбента из технологических колонок.

Исследование сорбции неодима перечисленными выше сорбентами показали, что значения динамических коэффициентов по неодиму для сорбента SBU-3 в 1.37 раза превышает аналогичную величину для сорбента AXIONIT MND 40T и 4.1 раза для BAU-1M (рис. 7).

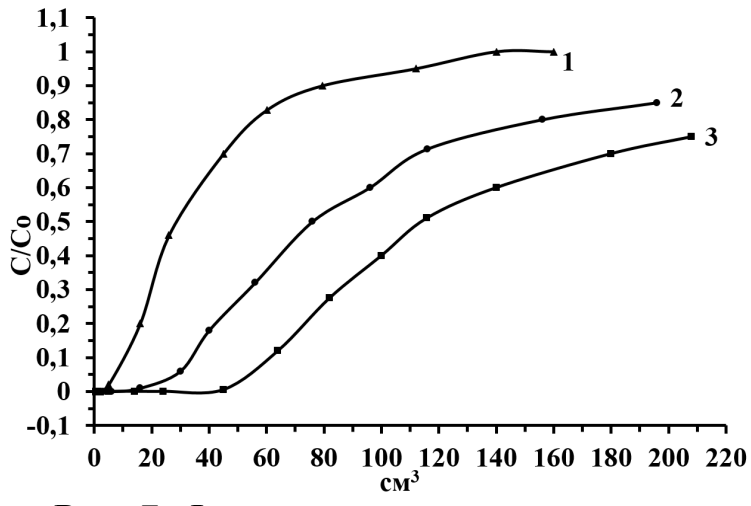

Рис. 7. Фронтальные выходные кривые для разных сорбентов, изготовленных на основе ТОДГА, при сорбции $\mathrm{Nd}$ $\left(4.6 \mathrm{мг} /\right.$ дм $\left.^{3}\right)$ из $1 \mathrm{M} \mathrm{HNO}_{3}$. 1-BAU-1M, 350 мг; 2-AXIONIT MND40T, 350 мг; 3- SBU-3, 350 мг

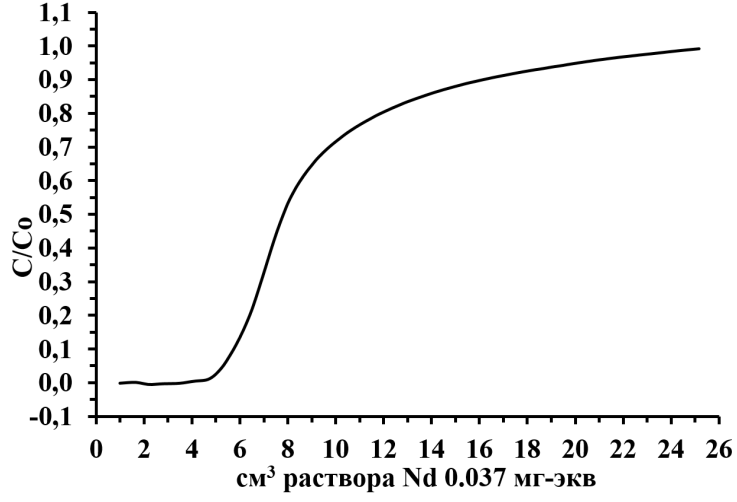

Рис. 8. Фронтальная кривая насыщения сорбента SBU-3. Масса сорбента в колонке 1.3 г; сорбция из 4М азотной кислоты; скорость потока $1 \mathrm{~cm}^{3} /$ мин. Детектирование по собственному спектру светопоглощения $\mathrm{Nd}$, при длине волны 794 нм

Динамическая емкость сорбента SBU-3 при сорбции неодима из 4M азотной кислоты до $10 \%$ проскока составляет 0.17 мг-экв/г. Величина полной динамической емкости по $\mathrm{Nd}$ составляет 0.71 мг-экв/г (рис. 8), что превышает величину 0.5 мгэкв/г, полученную для сорбента AXIONIT MND 40T. Дальнейшее исследование показало, что сорбированный неодим может быть практически количественно элюирован с сорбента SBU-3 водным раствором ДТПА в 0.05 М азотной кислоте (рис. 9).

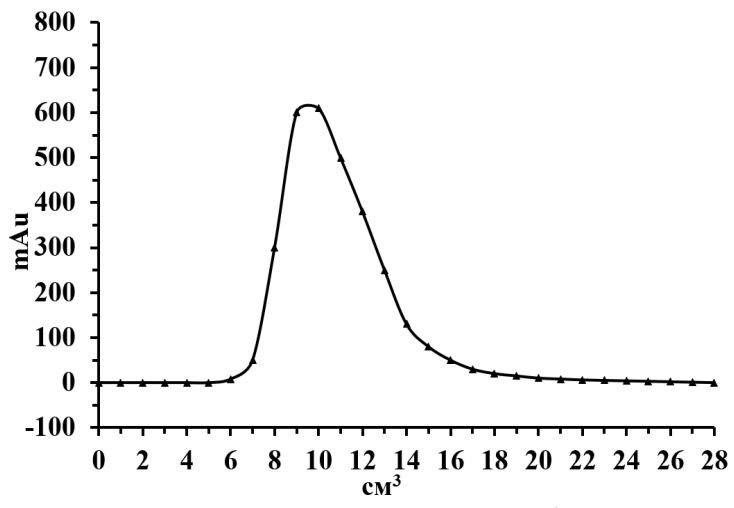

Рис. 9. Хроматограмма десорбции неодима водным раствором ДТПА (100 мг/дм ${ }^{3}$ ) в $0.05 \mathrm{M}$ азотной кислоте, 1350 мг сорбента SBU-3, 140 мкл, неодим 120 мг/дм ${ }^{3}$. Сорбция из $1 \mathrm{M} \mathrm{HNO}_{3}$ в течение 5 мин, затем десорбция водным раствором ДТПА. Скорость потока $1 \mathrm{~cm}^{3} /$ мин

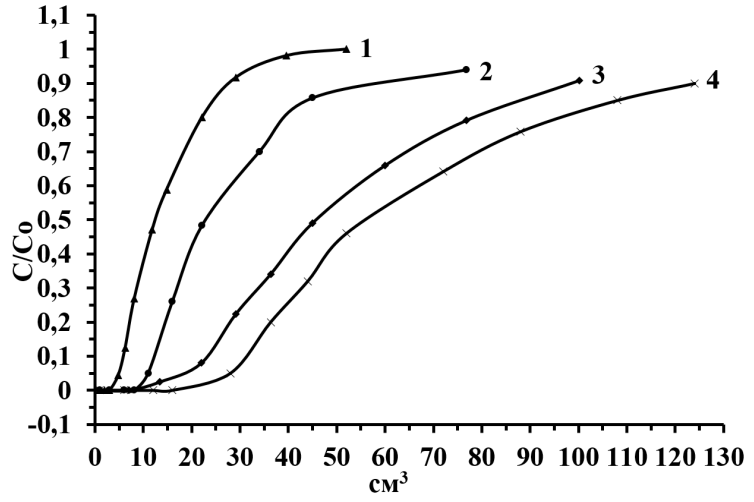

Рис. 10. Выходные кривые различных Р3Э при сорбции на сорбенте SBU-3 в $1 \mathrm{M}$ $\mathrm{HNO}_{3}$, скорость потока $1 \mathrm{~cm}^{3} /$ мин. 1-Лантан 50 мг/дм ${ }^{3}, 358$ мг сорбента; 2-Неодим 60 мг/дм 3 , 330 мг сорбента; 3-Самарий 70 мг/дм ${ }^{3}, 328$ мг сорбента; 4-Европий 80 мг/дм ${ }^{3}, 325$ мг сорбента 
Помимо неодима сорбент SBU-3 способен извлекать лантан, европий и самарий. На рисунке 10 представлены фронтальные выходные кривые для лантана, неодима, самария и европия, на основе которых были определены динамические коэффициенты разделения этих элементов $\left(\mathrm{K}_{\mathrm{d}}=\mathrm{V}_{0.5} / \mathrm{m}_{3}\right)$, анализ значений которых позволяет сделать вывод о возможности использования сорбента SBU-3 для внутригруппового разделения этих РЗЭ - значения динамических коэффициентов разделения для пар лантан /неодим, лантан/самарий и лантан /европий соответственно составляют $1.83,3.66$ и 4.58.

\section{Заключение}

Таким образом, проведенное исследование показывает высокий потенциал сорбентов импрегнированного типа на основе $\mathrm{N}, \mathrm{N}, \mathrm{N}^{\prime}, \mathrm{N}^{\prime}-$-тетраоктилдигликольамида для выделения неодима (а в перспективе - и америция) из азотнокислых сред. Сравнение сорбционных характеристик по неодиму для сорбента SBU-3, в котором в качестве матрицы использовался гидрофобизированный силикагель с аналогичными данными для других коммерчески доступных сорбентов российских производителей, позволяет сделать вывод о перспективности применения этого материала в практических технологиях.

Работа выполнена при частичной финансовой поддержке Программы фундаментальных научных исследований Президиума РАН №34 «Актуальные проблемь физикохимии поверхности и создания новых композитных материалов»

\section{Список литературы}

1. Аляпышев М.Ю., Бабаин В. А., Устынюк Ю.А. // Успехи химии. 2016. Т. 85. № 9 C. 943. DOI: https://doi.org/10.1070/RCR4589

2. Моходоева О.Б., Мясоедова Г.В., Захарченко Е.А. // Радиохимия. 2011. Т. 53. - № 1. C. 34-41. DOI: 10.1134/S106636221101005X

3. Sasaki Y., Sugo Y., Suzuki S., Tachimori S. // Solv. Extr. Ion Exch. 2001. Vol. 19. No 1. pp. 91-103. https://doi.org/10.1081/SEI100001376

4. Horwitz E.P., McAlister D.R., Bond A.H., Barrans R.E. // Solv. Extr. Ion Exch. 2005. Vol. 23. pp. 219-224. https://doi.org/10.1081/SEI200049898

5. Maxwell S.L., Culligan B.K. // J. of Radioanalytical and Nucl. Chem. 2006. Vol. 270.

\section{References}

1. Alyapyshev M.Yu., Babain V.A., Ustynyuk Yu.A., Uspekhi khimii, 2016, Vol. 85, No 9, pp. $943 . \quad$ DOI: https://doi.org/10.1070/RCR4589

2. Mokhodoeva O.B., Myasoedova G.V., Zakharchenko E.A., Radiokhimiya, 2011, Vol.
No 3. pp. 699-704. DOI https://doi.org/10.1007/s10967-006-0449-2

6. Ansari S.A., Pathak P.N., Husain M., Prasad A.K. et al. // Talanta. 2006. No 68. pp. 178180. DOI: $10.1016 /$ j.talanta.2005.07.042

7. Чухланцева Е.В., Сергеева Н.Л., Усолкин А.Н., Неудачина Л.К. и др. // Аналитика и контроль. 2012. Т. 16. № 2. С. 143-150. http://aik-

journal.urfu.ru/periodical/2012/2012_2.htm

8. Милютин В.В., Гелис В.М., Некрасова Н.А., Фирсова Л.А. и др. // Радиохимия. 2015. T. 57. № 5. C. 438-441. DOI: 10.1134/S1066362215050100

9. Браун Т., Герсини Г. Экстракционная хроматография. Пер. с англ. М. Мир. 1978. $628 \mathrm{c}$.

53, No 1, pp. 34-41. DOI: 10.1134/S106636221101005X

3. Sasaki Y., Sugo Y., Suzuki S., Tachimori S., Solv. Extr. Ion Exch., 2001, Vol. 19, No 1, pp. 91-103. https://doi.org/10.1081/SEI100001376 
4. Horwitz E.P., McAlister D.R., Bond A.H., Barrans R.E., Solv. Extr. Ion Exch., 2005, Vol. 23, pp. 219-224. https://doi.org/10.1081/SEI200049898

5. Maxwell S.L., Culligan B.K., J. of Radioanalytical and Nucl. Chem., 2006, Vol. 270, No 3, pp. 699-704. DOI https://doi.org/10.1007/s10967-006-0449-2

6. Ansari S.A., Pathak P.N., Husain M., Prasad A.K. et al., Talanta, 2006, No 68, pp. 178180. DOI: $10.1016 /$ j.talanta.2005.07.042

Баулин Владимир Евгеньевич - гл. н. с, д.х.н., лаборатории новых физико-химических проблем, ФГБУН Институт физической химии и электрохимии имени А.Н. Фрумкина, Москва, тел: (495) 724-36-60

Баулин Дмитрий Владимирович - инженер 1 категории лаборатории новых физикохимических проблем, ФГБУН Институт физической химии и электрохимии имени А.Н. Фрумкина, Москва

Усолкин Анатолий Николаевич - к.х.н, ведущий инженер-радиохимик ФГУП «ПО «Маяк», Озерск, Челябинская область

Ивенская Наталья Михайловна - инженертехнолог-химик-исследователь, ФГУП «ПО «Маяк», 456784, Озерск, Челябинская область

Власова Наталья Владимировна - инженер-технолог-химик-исследователь, «ПО «Маяк», 456784, Озерск, Челябинская область

Козлов Павел Васильевич - руководитель группы центральной заводской лаборатории, ФГУП «ПО «Маяк», Озерск, Челябинская область

Ремизов Михаил Борисович - начальник технологической лаборатории по обращению с РАО, ФГУП «ПО «Маяк», Озерск, Челябинская область

Чухланцева Екатерина Владимировна руководитель группы центральной заводской лаборатории, ФГУП «ПО «Маяк», Озерск, Челябинская область

Цивадзе Аслан Юсупович - академик РАН, научный руководитель ФГБУН Институт физической химии и электрохимии имени А.Н. Фрумкина, Москва
7. Chukhlantseva E.V., Sergeeva N.L., Usolkin A.N., Neudachina L.K. et al., Analitika $i$ kontrol', 2012, Vol. 16, No 2, pp. 143-150. http://aik-

journal.urfu.ru/periodical/2012/2012 2.htm

8. Milyutin V.V., Gelis V.M., Nekrasova N.A., Firsova L.A. et al., Radiokhimiya, 2015, Vol. 57, No 5, pp. 438-441. DOI: 10.1134/S1066362215050100

9. Braun T., Gersini G. Ekstraktsionnaya khromatografiya. Per. s angl., M., Mir, 1978, $628 \mathrm{p}$.

Baulin Vladimir E. - principal scientific collaborator of the Laboratory of New Physicochemical Problems, Russian academy of sciences A.N. Frumkin Institute of Physical chemistry and Electrochemistry RAS (IPCE RAS), Moscow, Russia, e-mail: mager1988@gmail.com

Baulin Dmitrii V. $-1^{\text {st }}$ category Engineer of the Laboratory of New Physicochemical Problems, Russian academy of sciences A.N. Frumkin Institute of Physical chemistry and Electrochemistry RAS (IPCE RAS), Moscow, Russia

Usolkin Anatolii N. - PhD, leading engineer- radiochemist. FSUE «PA «Mayak», Ozersk, Chelyabinsk region

Ivenskaya Natalia M. - engineer-technologistchemist-researcher, FSUE «PA «Mayak», Ozersk, Chelyabinsk region

Vlasova Natalia V. - engineer-technologistchemist-researcher, FSUE «PA «Mayak», Ozersk, Chelyabinsk region

Kozlov Pavel V. - head of group of Central Plant Laboratory, FSUE «PA «Mayak», Ozersk, Chelyabinsk region

Remizov Michail B. - head of laboratory for Radioactive Waste Management, FSUE «PA «Mayak», 456784, Ozersk, Chelyabinsk region

Chuchlanseva Ekaterina V. - head of group of Central Plant Laboratory, FSUE «PA «Mayak», Ozersk, Chelyabinsk region

Tsivadze Aslan Yu. - research supervisor of the Laboratory of New Physicochemical Problems, Russian academy of sciences A.N. Frumkin Institute of Physical chemistry and Electrochemistry RAS (IPCE RAS), 31 Leninsky prospect, Moscow 